\title{
Photochromism and Thermally and Optically Stimulated Luminescences of AIN Ceramic Plate for UV Sensing
}

\author{
Go Okada, ${ }^{*}$ Takumi Kato, Daisuke Nakauchi, Kentaro Fukuda, ${ }^{1}$ and Takayuki Yanagida \\ Graduate School of Materials Science, Nara Institute of Science and Technology (NAIST), \\ 8916-5 Takayama, Ikoma, Nara 630-0192, Japan \\ ${ }^{1}$ Tokuyama Corporation, 1-1 Mikage-cho, Shunan-shi, Yamaguchi 745-8648, Japan
}

(Received January 7, 2016; accepted April 13, 2016)

Keywords: AlN, photochromism, TSL, OSL, UV sensing, imaging

We have characterized AlN ceramic plates (SHAPAL ${ }^{\circledR}$, Tokuyama Corporation, Japan) for UV sensing applications. The SHAPAL ${ }^{\circledR}$ AlN ceramic plate is a commercially available product used as a heat sink, but it shows photochromic effects and thermally and optically stimulated luminescences (TSL and OSL) after it is exposed to UV light. Upon UV exposure, a broad optical absorption band is induced in the UV-blue region $(2.5-4.5 \mathrm{eV})$, and the color changes from gray to orange. A UVexposed AlN ceramic plate emits near UV light $(\sim 3.4 \mathrm{eV})$ upon thermal or optical stimulation. The TSL signal showed maximum intensity when heated to around $200{ }^{\circ} \mathrm{C}$, whereas the OSL could be stimulated by light exposure over a wide spectral range from $\sim 2$ to $3 \mathrm{eV}$. The sensitivity of the photochromism, TSL, and OSL depended on the UV photon energy. Overall, these UV-induced effects were strongly pronounced for photon energies higher than $4.0 \mathrm{eV}$, but the photochromic effect could be seen for lower energies, though the signal was considerably smaller. The AlN ceramic plate could be used for UV sensing multiple times. The photochromic, TSL, and OSL signals could be erased by heat treatment, and the OSL signal could also be erased by exposure to low-energy light.

\section{Introduction}

UV sensing techniques are of considerable importance with a wide range of applications including homeland security and forensics, ${ }^{(1,2)}$ and biology. ${ }^{(3,4)}$ There are different approaches to measuring the two-dimensional distribution of UV light or UV images. One approach is to use a recording medium that records the distribution of UV light intensity in a two-dimensional area in the form of a UV-induced effect. An example of such a medium is photographic film. Another approach is to use a combination of a phosphor plate with a conventional photodetector. ${ }^{(5)}$ The phosphor plate converts UV light to visible light so that a conventional photodetector can indirectly detect the signal. Furthermore, with considerable recent advancements of semiconductor technologies, electronic semiconductor arrays such as charge-coupled device (CCD) and complementary metal-oxide-semiconductor (CMOS) have been developed for UV sensing so that UV light can be directly converted to electronic signals. ${ }^{(6,7)}$ These different approaches have advantages and disadvantages. For example, UV-recording media are typically inexpensive,

"Corresponding author: e-mail: go-okada@ms.naist.jp 
but they do not allow real-time measurements. Also, film-based techniques require developing processes that are time-consuming. The combination of a phosphor plate and a photodetector makes real-time measurements possible, but considerable light scattering at the phosphor layer suppresses image resolution. Because direct measurement using a semiconductor-based imaging sensor does not involve such optical-based readout process, the image resolution is significantly improved. However, the image resolution of such a semiconductor-based imaging sensor is limited by pixel size, or semiconductor fabrication technologies, and such detectors tend to be expensive to make.

AlN is of current interest for UV sensing applications. In general, AlN is used for UV-emitting light-emitting diodes (LEDs) for direct transitions over the wide optical bandgap of AlN $(\sim 6.2 \mathrm{eV}){ }^{(8)}$ Also, because of its high thermal conductivity and electrical insulation, it is used as a heat sink. Earlier, we reported that an AlN ceramic plate (SHAPAL ${ }^{\circledR}$, Tokuyama Corporation), which is commercially available for use as a heat sink, can also be used in radiation sensing applications. ${ }^{(9-11)}$ It has been shown that AlN ceramic plates show thermally stimulated luminescence (TSL) and optically stimulated luminescence (OSL) upon exposure to X-rays. TSL and OSL are well-known phenomena and often used for measuring ionizing radiation. ${ }^{(12-15)}$ In this work, however, we have found that AlN ceramic platea are also sensitive to UV light and show photochromism, TSL, and OSL effects upon UV exposure. Earlier studies have reported that some inorganic solids have shown UV-induced photochromism ${ }^{(16)}$ and storage phosphor properties; ${ }^{(17,18)}$ however, as far as we are aware, there are not many materials reported to show both of these phenomena. Unlike the properties of photographic films, these UV-induced effects do not require post-treatment such as a developing process. Furthermore, using optical signals as a measure of UV light has a strong advantage in that they can be used with optical microscopes for high-resolution imaging. The aim of this work is to study these UV-induced effects on the SHAPAL ${ }^{\circledR}$ AlN ceramic plate comprehensively and to extend our understanding of these phenomena for potential UV sensing applications. Our preliminary experimental results are shown in this paper.

\section{Materials and Methods}

The AlN ceramic plates used in this research were produced by Tokuyama Corporation, Yamaguchi, Japan: they are commercially available for use as heat sinks. The available sample size was as large as $\sim 120 \times 120 \mathrm{~mm}^{2}$ with a thickness of $\sim 0.65 \mathrm{~mm}$. The following characterizations were carried out at the Nara Institute of Science and Technology (NAIST), Nara, Japan. The optical diffuse transmittance was measured using a Shimadzu spectrophotometer (SolidSpec-3700). For the TSL measurement, a sample was placed on a ceramic heater while the temperature was controlled by a SAKAGUCHI temperature controller (SCR-SHQ-A). The TSL emission spectrum and relative intensity were then measured using a CCD-based spectrometer (QEPro, Ocean Optics). A Hamamatsu Quantaurus-Tau (C11367) was used to measure the OSL emission spectrum with stimulation at $1.97 \mathrm{eV}(630 \mathrm{~nm})$; the OSL stimulation spectrum as well as OSL response curve, in which the OSL signal is collected as a function of time during stimulation light exposure, were measured using a spectrofluometer (FP-8600, JASCO). The xenon light source in the latter instrument and a 4.9-eV (254-nm) mercury lamp (3UV-34, UVP) were used as UV sources. The former was used to study spectral dependence of UV-induced phenomena, while the latter was used for other general purposes. 


\section{Results and Discussion}

The type of AlN ceramic plate $\left(\mathrm{SHAPAL}^{\circledR}\right)$ sample used in this research is illustrated in Fig. 1(a). The sample is $59 \times 38 \mathrm{~mm}^{2}$ with a thickness of $0.65 \mathrm{~mm}$. Owing to the nature of the ceramic sintering process, the plate can be easily made in any form homogeneously. Homogeneity is one of the most important features of a two-dimensional imaging plate, because it allows for the measurement of spatial distribution. For UV sensing applications, we have characterized the photochromism, TSL, and OSL properties of SHAPAL ${ }^{\circledR}$ AlN ceramic plates, and discussed them in this section.

\subsection{Photochromism}

As illustrated in Fig. 1, the as-provided AlN ceramic plate was gray. However, once it was exposed to UV light, the color changed to orange. Figure 1(b) shows a comparison of sample plates exposed to UV light $(\sim 4.9 \mathrm{eV})$ for different times. It is clearly seen that the extent of coloration increases with exposure time.

Figure 2(a) shows the optical absorbance of an AIN ceramic plate induced by UV exposure. Here, the induced absorbance was derived as

$$
\begin{aligned}
A_{\mathrm{i}}(\lambda) & =A_{\mathrm{UV}}(\lambda)-A_{\mathrm{o}}(\lambda) \\
& =-\ln \left(T_{\mathrm{UV}}(\lambda)\right)+\ln \left(T_{\mathrm{o}}(\lambda)\right),
\end{aligned}
$$

where $A_{\mathrm{i}}(\lambda)$ is the UV-induced absorbance, $A_{\mathrm{UV}}(\lambda)$ and $A_{\mathrm{o}}(\lambda)$ are the absorbances after and before UV exposure, and $T_{\mathrm{UV}}(\lambda)$ and $T_{\mathrm{o}}(\lambda)$ are the transmittances after and before UV exposure. As seen in the figure, optical absorption was strongly induced by UV exposure in the range of $2.5-4.5 \mathrm{eV}$. Above $4.5 \mathrm{eV}$, the measurement was accurate because the measured transmittance signal was very small in this range. Below $2.0 \mathrm{eV}$, an induced absorption band was present but it was very weak compared to the dominant absorption at around $3 \mathrm{eV}$. The induced absorbance may be approximated by a combination of Gaussian functions. The least-square curve fitting technique was applied here, and

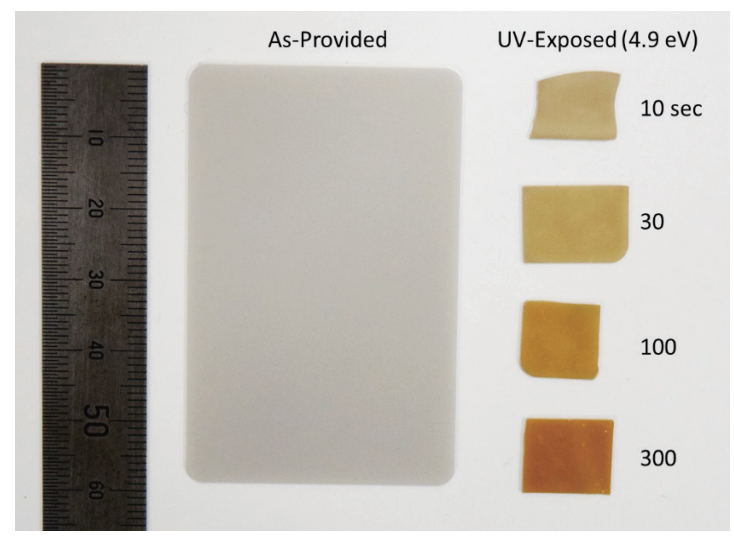

(a)

(b)

Fig. 1. (Color online) AlN ceramic plate $\left(\mathrm{SHAPAL}^{\circledR}\right.$, Tokuyama Corporation, Japan) sample as provided and those exposed to UV light $(\sim 4.9 \mathrm{eV})$ for different times $(10,30,100$, and $300 \mathrm{~s})$. 


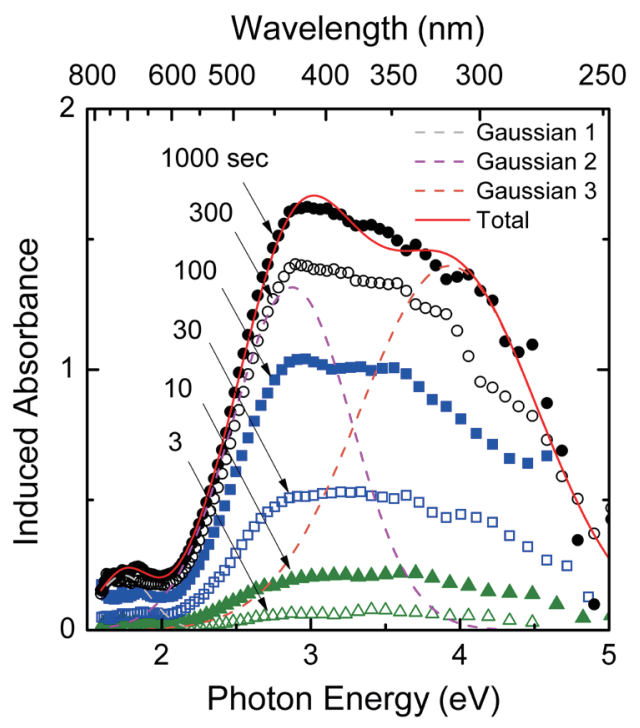

(a)

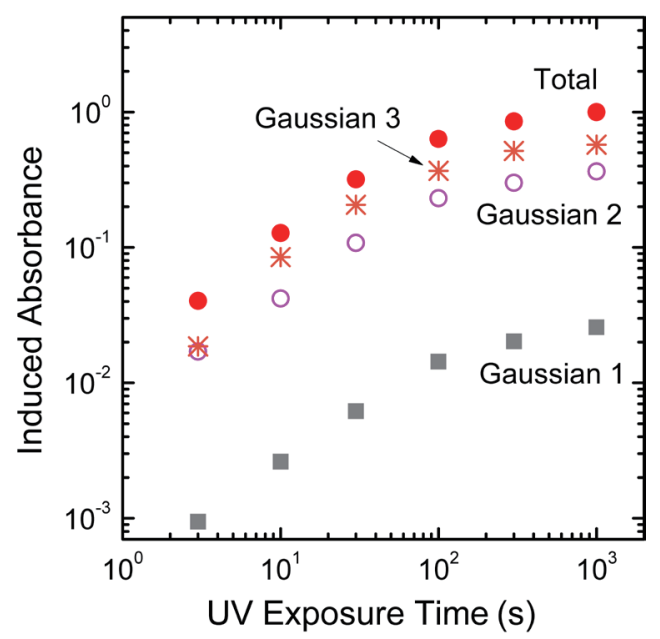

(b)

Fig. 2. (Color online) UV-induced optical absorbance spectra for different UV exposure times (a), and integrated absorbance as a function of exposure time (b).

the result suggested that there were at least three absorption bands present with center positions at 1.8, 2.9, and $3.8 \mathrm{eV}$. Figure 2(b) illustrates the induced absorbance as a function of UV exposure time. The absorbance increased linearly with the exposure time up to several tens of seconds; however, it seemed to saturate at longer exposure times. This is a common phenomenon, and it is understood that color centers are generated by trapping charges (electrons or holes) at localized trapping centers and that they possess the ability to absorb light. Our experimental results suggest that the charge generation and trapping process is proportional for UV exposures shorter than several tens of seconds, while the generation of additional centers becomes inefficient for longer exposures and eventually saturates.

\subsection{TSL}

After an AlN sample was exposed to UV light, it showed TSL as it was heated at elevated temperatures. Figure 3 shows a summary of some of the TSL properties measured on a SHAPAL ${ }^{\circledR}$ AIN ceramic plate. As seen in Fig. 3(a), it exhibits TSL emission very broad features in both the spectrum and glow temperature curve. The emission spectrum [Fig. 3(c)] has a peak at around $3.3 \mathrm{eV}$. As soon as the sample was heated at elevated temperatures, the TSL signal appeared and reached its maximum value at around $200{ }^{\circ} \mathrm{C}$ when heated at a rate of $1{ }^{\circ} \mathrm{C} / \mathrm{s}$. The signal then decreased at higher temperatures. At $400{ }^{\circ} \mathrm{C}$, the TSL signal is completely suppressed. The glow curve seems to have decomposed into two Gaussians with peaks at 130 and $213{ }^{\circ} \mathrm{C}$ as shown in Fig. 3(b). The latter fact suggests that there are at least two different charge trapping centers involved in the TSL process. Figure 3(d) illustrates that the TSL response intensity increases with increasing UV exposure time up to $100 \mathrm{~s}$, which is then followed by saturation. The occurrence of saturation seems to take place approximately at the same exposure time as that of photochromism (and OSL which is described below). Here, the TSL response used is the integrated signal over 


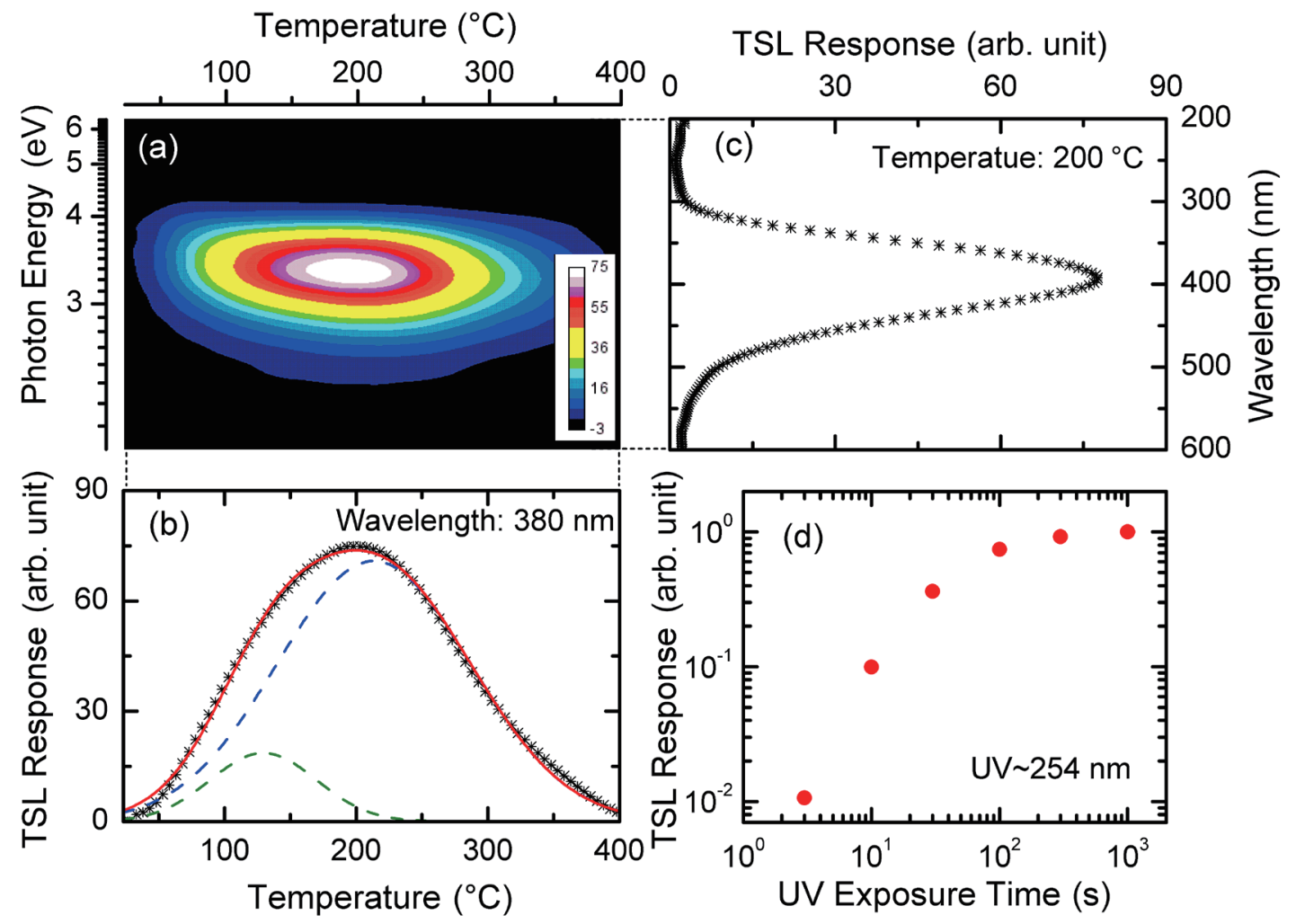

Fig. 3. (Color online) Contour graph of TSL emission spectrum (a) as a function of heating temperature, (b) the corresponding glow curve monitoring at $380 \mathrm{~nm}$, (c) the TSL emission spectrum monitoring at $200{ }^{\circ} \mathrm{C}$, and (d) the TSL response as a function of UV exposure time. The heating rate was $1{ }^{\circ} \mathrm{C} / \mathrm{s}$.

the temperature range measured. It is also worth mentioning here that the spectral peak position of TSL emission slightly shifts towards a lower energy (longer wavelength) by the UV exposure (data not shown) owing to the influence of an induced absorbance band in the UV region, which was discussed in the previous section. Since the peak position is also dependent on the UV exposure time, it can also be used as a probe for sensing.

\subsection{OSL}

After a sample was exposed to UV light, OSL emission could be observed when stimulated by visible light. Figure 4(a) shows the OSL emission and stimulation spectra. The OSL emission band is located at $3.4 \mathrm{eV}$ with an approximately $0.5 \mathrm{eV}$ full-width at half-maximum (FWHM). The latter OSL emission could be stimulated by exposure to light of wide energy range but low emission wavelength. Accepting the analogy of the charge trapping process described in the previous paragraph, the trapped charges could be released by external optical stimulation, and then the freed charges could recombine at the emission center, which could result in the OSL emission of 3.4 $\mathrm{eV}$. Determining the origin of emission centers as well as charge trapping centers is beyond the scope of this study. However, we speculate that some defect centers played a role, as no intentional impurities were included. As shown in Fig. 4(b), the saturation of the OSL signal takes place at 


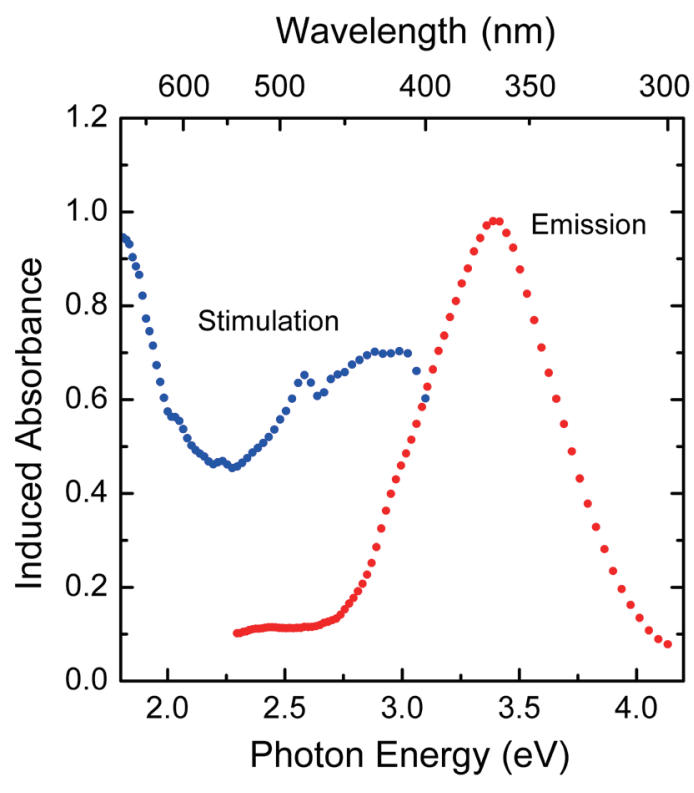

(a)

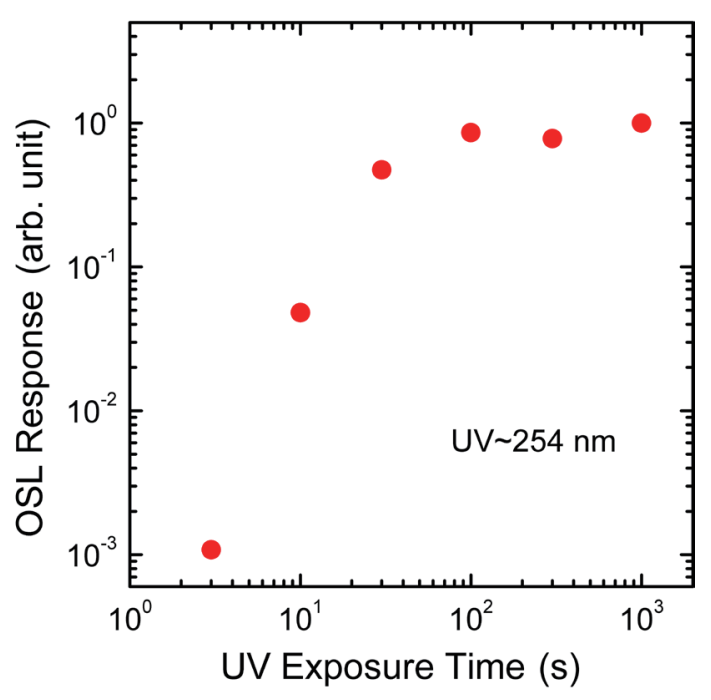

(b)

Fig. 4. (Color online) OSL emission and stimulation spectra of AIN ceramic plate (a) and the OSL response as a function of UV exposure time (b).

around $100 \mathrm{~s}$ and above, which is approximately the same range as those of the photochromic effect and TSL. This suggests that these three UV-induced phenomena are somewhat related each other. In addition, as observed in TSL, the peak position of OSL emission shifts towards lower energy (data not shown) owing to the UV-induced optical absorption. The peak position can also be used as an indication of UV dose for sensing applications.

\subsection{Spectral response}

It has been shown in a previous section that the AlN ceramic plate was sensitive to UV light and displayed photochromism, TSL, and OSL responses as a result of UV exposure. These responses depended on the energy of the exposed photons. Figure 5 depicts the photochromism, TSL, and OSL responses as a function of the energy of the exposing photons. Here, for the measurements of these responses, the total intensity of light exposure was fixed, while the photon flux was varied owing to the spectral distribution of the light source. The heating rate used for the TSL measurement was $1{ }^{\circ} \mathrm{C} / \mathrm{s}$. The light for stimulating OSL was $2 \mathrm{eV}$. Overall, the responses were considerably small in the range of 3.0-4.0 eV. However, as the photon energy was increased, the responses increased dramatically above $4.0 \mathrm{eV}$ and reached peak values at around $4.8 \mathrm{eV}$, which was followed by a slight decrease at higher photon energies. Despite these similarities between photochromism, TSL, and OSL responses, the sharp increase seemed to take place at an energy lower by approximately $0.2 \mathrm{eV}$ for photochromism than for TSL and OSL. Furthermore, it seemed that some photochromic response, though small, was present in the low energy range. These observation indicated that the mechanisms of photochromism caused by UV exposure was different from those of other responses. 


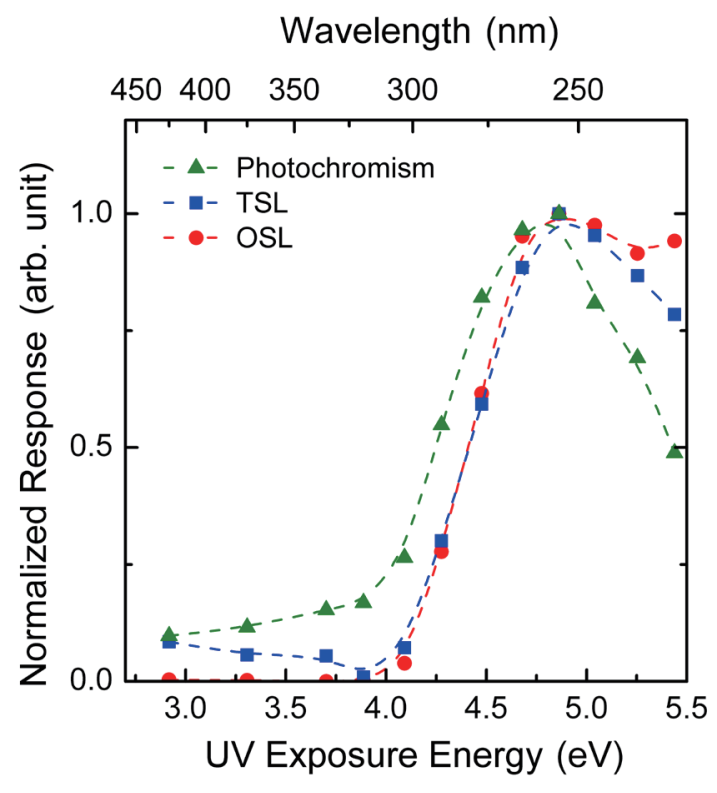

Fig. 5. (Color online) Spectral response of photochromism, TSL, and OSL in AlN ceramic plate.

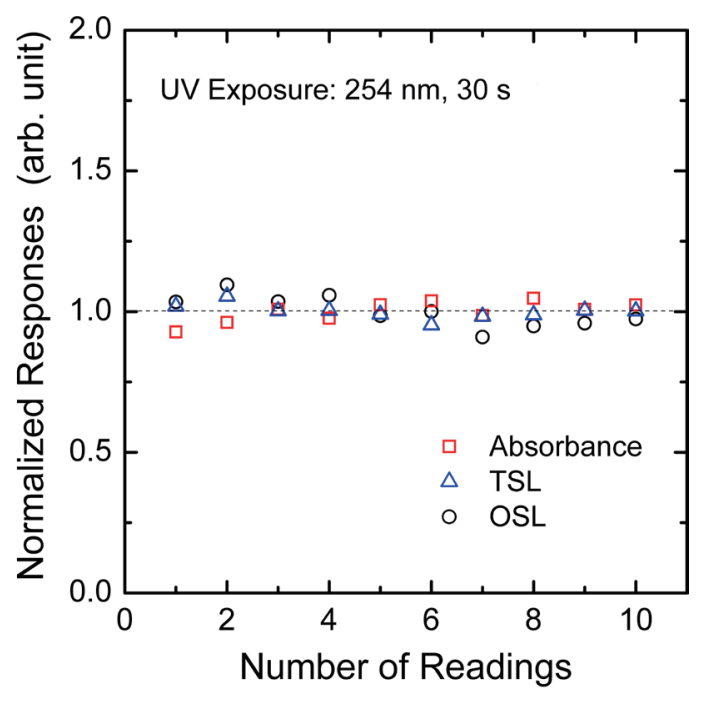

Fig. 6. (Color online) Photochromic, TSL, and OSL responses of a SHAPAL ${ }^{\circledR}$ AlN ceramic plate as a function of the number of readings. For the absorbance measurement, the sample was annealed at $900{ }^{\circ} \mathrm{C}$ for $5 \mathrm{~min}$ to erase the recorded value before each UV exposure. The UV exposure was carried out at $254 \mathrm{~nm}$ for $30 \mathrm{~s}$.

This observation is consistent with the transmittance measurement (data not shown), which indicates a significant absorption band in the range above $4 \mathrm{eV}$. The strong optical absorption results in photoionization, and the charges generated are subsequently trapped at localized sites. Note here that the optical bandgap of AlN is $\sim 6.2 \mathrm{eV} .^{(8)}$ The latter energy is much higher than the absorption edge observed in our measurements. For these reasons, we think that the photoionization involved in our case is not caused by photoabsorption due to electronic transitions from the valence band to the conduction band. Rather, our observation indicates that the photoionization process should be assisted by other bands which are possibly present owing to the inclusion of some defects and unintentional impurities; it could also be assisted by thermal ionization processes in excited states. ${ }^{(19)}$

\subsection{Reusability}

The SHAPAL ${ }^{\circledR}$ AlN ceramic plate is reusable as a UV sensor. Figure 6 illustrates multiple uses of an AIN ceramic plate as a UV sensor using photochromism, TSL, and OSL phenomena over 10 times. For the absorbance measurements, as a response of the photochromic effect, the sample was annealed before each UV exposure to erase the value recorded by the previous measurement. On the other hand, the TSL and OSL measurements does not require any particular treatment to erase the signal besides the signal reading process. The experimental results clearly demonstrate that these UV-induced phenomena are, within the accuracy of measurement, well-reproduced multiple times, and hence that the AlN ceramic plate is reusable as a UV sensor. 


\section{Conclusions}

In this work, we have established for the first time that SHAPAL ${ }^{\circledR}$ AlN ceramic plates (Tokuyama Corporation, Japan) show photochromic, TSL, and OSL responses as a result of UV exposure. The AlN ceramic plate was originally grey as prepared, but turned orange once exposed to UV light. The induced optical absorption was significant over a photon energy range of 2-4 eV. The absorption intensity increased with the extent of UV exposure. After the UV exposure, the SHAPAL ${ }^{\circledR}$ AlN ceramic plate also showed OSL effects. After exposure to light with a lower energy, OSL emission could be observed at around $3.4 \mathrm{eV}$. The OSL signal depended on the energy of the exposing UV photons. The response was very sensitive to UV light higher than $4 \mathrm{eV}$. The OSL response was repeatable multiple times (tested 10 times in this work); therefore, the SHAPAL ${ }^{\circledR}$ AlN ceramic plate is reusable as a UV sensing plate. Such a fluorescence imaging plate may be of great interest for sub-micrometer imaging applications. ${ }^{(20)}$

\section{Acknowledgements}

This research was co-supported by a Grant-in-Aid for Scientific Research (A) (26249147), a Grant-in-Aid for Research Activity start-up (15H06409), and Green Photonics Research from the Ministry of Education, Culture, Sports, Science and Technology of the Japanese government (MEXT). It was also partially supported by the Adaptable and Seamless Technology Transfer Program (A-STEP) by the Japan Science and Technology (JST) Agency, the Murata Science Foundation, Hitachi Metals/Materials Science Foundation, and a cooperative research project of the Research Institute of Electronics, Shizuoka University.

\section{References}

1 R. E. Barsley, M. H. West, and J. A. Fair: Am. J. Forensic Med. Pathol. 11 (1990) 300.

2 A. Richards and R. Leintz: J. Forensic Identif. 63 (2013) 46.

3 J. F. Hassard, S. Hassard, and A. M. Mainwood: US Patent No. 6,017,435 (2000).

4 F. Ye, A. Yaghmur, H. Jensen, S. W. Larsen, C. Larsen, and J. Østergaard: Eur. J. Pharm. Sci. 43 (2011) 236.

5 W. A. R. Franks, M. J. Kiik, and A. Nathan: IEEE Trans. Electr. Devices 50 (2003) 352.

6 H. Ishihara, S. Sugio, and T. Kanno: Sens. Mater. 22 (2010) 357.

7 C.-W. Chen, C.-C. Huang, Y.-Y. Lin, W.-F. Su, L.-C. Chen, and K.-H. Chen: Appl. Phys. Lett. 88 (2006) 073515.

8 Y. Taniyasu, M. Kasu, and T. Makimoto: Nature 441 (2006) 325.

9 T. Yanagida, Y. Fujimoto, N. Kawaguchi, and S. Yanagida: J. Ceram. Soc. Jpn. 121 (2013) 988.

10 G. Okada, K. Fukuda, S. Kasap, and T. Yanagida: 3rd Int. Work. Persistent Photostimulable Phosphors, Nov. 9-13, Arlington, USA (2015).

11 G. Okada, K. Fukuda, and T. Yanagida: 2015 IEEE Nucl. Sci. Symp. Oct. 31-Nov. 7, San Diego, USA (2015).

12 H. Nanto, R. Nakagawa, T. Yanagida, and Y. Fujimoto: Sens. Mater. 27 (2015) 277.

13 H. Masai, T. Yanagida, and T. Fujiwara: Sens. Mater. 27 (2015) 237.

14 Y. Fujimoto, T. Yanagida, M. Koshimizu, and K. Asai: Sens. Mater. 27 (2015) 263.

15 T. Yanagida, Y. Fujimoto, K. Watanabe, and K. Fukuda: Radiat. Meas. 6 (2014) 1.

16 J. Ueda, T. Shinoda, and S. Tanabe: Opt. Mater. Express 3 (2013) 787.

17 H. Nanto, F. Endo, Y. Hirai, S. Nasu, S. Taniguchi, and N. Takeuchi: J. Appl. Phys. 75 (1994) 7493.

18 H. Nanto, Y. Hirai, M. Ikeda, M. Kadota, J. Nishishita, S. Nasu, and Y. Douguchi: Sens. Actuators, A 53 (1996) 223.

19 J. Ueda, S. Tanabe, and T. Nakanishi: J. Appl. Phys. 110 (2011) 053102.

20 G. Okada, B. Morrell, C. Koughia, A. Edgar, C. Varoy, G. Belev, T. Wysokinski, D. Chapman, and S. Kasap: Appl. Phys. Lett. 99 (2011) 121105. 